\title{
Forward Modelling the O3(a+b) GW transient mass distributions with BPASs by varying compact remnant mass and SNe kick prescriptions
}

\author{
Sohan Ghodla ${ }^{1}$, Wouter G. J. van Zeist ${ }^{1}$, J. J. Eldridge ${ }^{\star}$, Héloïse F. Stevance ${ }^{1}$, \\ Elizabeth R. Stanway ${ }^{2}$ \\ ${ }^{1}$ Department of Physics, University of Auckland, Private Bag 92019, Auckland, New Zealand \\ ${ }^{2}$ Department of Physics, University of Warwick, Gibbet Hill Road, Coventry, CV4 7AL, UK
}

Accepted XXX. Received YYY; in original form ZZZ

\begin{abstract}
We present forward modelling from the BPAss code suite of the population of observed gravitational wave $(\mathrm{GW})$ transients reported by the LIGO/VIRGO consortium (LVC) during their third observing run, O3(a+b). Specifically, we predict the expected chirp mass and mass ratio distributions for GW transients, taking account of detector sensitivity to determine how many events should have been detected by the current detector network in $\mathrm{O} 3(\mathrm{a}+\mathrm{b})$. We investigate how these predictions change by alternating between four different remnant mass estimation schemes and two supernovae ( $\mathrm{SNe}$ ) kick prescriptions. We find that none of the model populations resulting from these variations accurately match the whole O3(a+b) GW transient catalog. However, agreement from some models to part of the catalog suggests ways to achieve a more complete fit. These include reducing the number of low mass black holes (BHs) close to the mass gap, while also increasing the number of higher mass BHs below the pair-instability SN limit. Finally, we find that the interaction between the value of the remnant mass from a stellar model and the choice of SN kick is complex and different kick prescriptions may be required depending on whether a neutron star or $\mathrm{BH}$ is formed.
\end{abstract}

Key words: stars: evolution - binaries: general - supernovae: general - methods: numerical

\section{INTRODUCTION}

In the span of only almost half a decade, the detection of GW transients has become almost routine. After LVC O3(a+b), there are now $90^{1}$ known GW transients events with a probability of being a real astrophysical signal $\left(p_{\text {astro }}\right)>50 \%$ (Abbott et al. 2020a; The LIGO Scientific Collaboration et al. 2021b). Each event in itself reveals much about the evolution of binary stars (e.g. for individual studies see Abbott et al. (2016, 2017, 2020b) and for evolutionary implications for binaries, see Eldridge \& Stanway (2016); Belczynski et al. (2016); Stevenson et al. (2017); Mapelli et al. (2017); Vigna-Gómez et al. (2018); Eldridge et al. (2019); Lipunov et al. (2021); Broekgaarden et al. (2021) and references therein). However with a growing understanding of the distribution of masses, we are now beginning to constrain the population of GW transients both through parametric models (e.g. Abbott et al. 2019; Abbott et al. 2020a; The LIGO Scientific Collaboration et al. 2021b) and using the technique of population synthesis to under-

\footnotetext{
^ j.eldridge@auckland.ac.nz

1 Overall, 11 events from $\mathrm{O} 1+\mathrm{O} 2,44$ events from $\mathrm{O} 3 \mathrm{a}$ and 35 events from $\mathrm{O} 3 \mathrm{~b}$
}

stand the physical process of stellar evolution that gives rise to the mass distribution (e.g. Belczynski et al. (2008); Stevenson et al. (2017); Eldridge et al. (2017); Giacobbo et al. (2018) and references therein).

While binary population synthesis is a powerful tool, there are many uncertainties in the physics incorporated in these models (e.g. De Marco \& Izzard 2017) as well as in the prescriptions used to model the star formation history and chemical evolution of the Universe (Belczynski et al. 2016; Mapelli et al. 2017; Chruslinska et al. 2019; Tang et al. 2020; Boco et al. 2021; Broekgaarden et al. 2021). In particular, apart from the uncertainties in evolution of massive single stars (eg. mass-loss rate, rotation, overshooting, etc.) the understanding of the mass-transfer and common envelope phase in binaries is also incomplete (e.g., Ivanova et al. 2013 and references therein). In addition, though the delaytime distribution is believed to mostly lie within 0-4.5 Gyrs (e.g. Fishbach \& Kalogera 2021), a subset of GW mergers has also been found to come from very long time delays (e.g., Eldridge \& Stanway 2016; Mapelli et al. 2017). As such, the wide distribution of possible delaytime intervals between the formation of the progenitor stellar binary of a GW transient and the inspiral event introduces sensitivity to past star formation conditions. Another required assumption is the remnant mass 


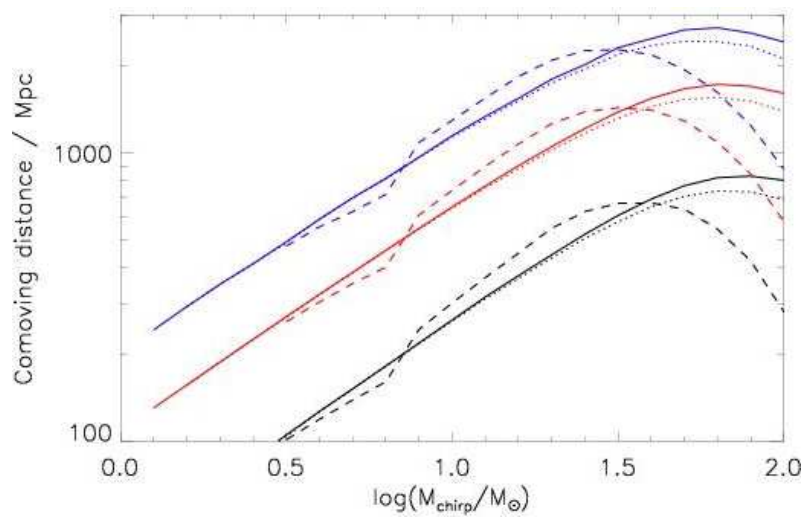

Figure 1. The detection distance versus chirp mass as a function of detection probability and mass ratio. The solid, dotted and dashed lines represent the mass ratio values $q=1, q=0.5$ and $q=0.1$. The black lines are for a detection probability of 0.9 , red 0.5 and blue 0.1 .

prescription used to determine the compact remnant mass once the stellar model reaches its final state, which often does not proceed all the way to core-collapse for numerical reasons. To this end, several remnant mass prescriptions with different features also exist in the literature, e.g. see, Patton \& Sukhbold (2020).

In this letter, we utilize BPAss (Binary Population and Spectral Synthesis) code results to forward model the population of GW transients resulting from their underlying stellar population up till their chirp event, specifically predicting the distribution of expected chirp masses and mass ratios for them during LVC O3(a+b). Section 2 outlines the numerical model, highlighting the differences between this and earlier BPAss work. Specifically, here we vary two key uncertainties in our analysis, (i) the nature of the kick that remnants receive while undergoing a $\mathrm{SN}$ explosion and (ii) the prescription for calculating the resulting remnant masses. Section 3 presents our results on the number of expected detections and the predicted GW transient object mass distribution. We then compare these to the LVC O3 $(a+b)$ catalog (The LIGO Scientific Collaboration et al. 2021b) to see which of the prescriptions produce a mass distribution that closely matches their results, presenting discussion and concluding remarks in section 4 .

\section{METHODS AND PRESCRIPTIONS, SIMULATIONS AND OBSERVATIONS}

Our aim here is to forward model the population of GW transients that may have been detected in $\mathrm{O} 3(\mathrm{a}+\mathrm{b})$ (henceforth $\mathrm{O} 3$ ), making predictions independent of the $\mathrm{O} 3$ observations, and explore some of the stellar evolutionary uncertainties that might impact these results. We use four different schemes to estimate the remnant masses resulting from stellar evolution and two different kicks to investigate how they collectively affect the populations of the predicted GW mergers.

\subsection{Population synthesis}

We use BPASS v2.2.1 stellar models (Eldridge et al. 2017; Stanway \& Eldridge 2018) and build upon our earlier work on predicting the GW transient rates. We refer the reader to those papers for full details of our model and method (Eldridge \& Stanway 2016; Eldridge et al. 2019; Tang et al. 2020). We use the same underlying model of the cosmological star formation history and metallicity evolution as in Eldridge et al. (2019). However, two aspects of our previous population synthesis have been improved.

Firstly, we have updated the treatment of rejuvenation during mass transfer onto the secondary stars. BPASs has two principal sets of models: primary models where the more massive star's full structure is computed in detail and the secondary's evolution is approximated using the equations of Hurley et al. (2000); and secondary models where the secondary is evolved in detail as either a single star or a star in orbit around a compact object - depending on the outcome of the first SN. Many different primaries map onto the same secondary model. As a result, in earlier BPAss calculations we used an average rejuvenation age (i.e. time step at which mass transfer leads to rotational mixing, causing the accretor to become chemically homogeneous eg., Maeder 1987) for each secondary model. In this work, we use a distribution of rejuvenation ages for each secondary model. This has the effect of smoothing out our delaytime distribution for transient events driven by GW emission.

Secondly, we now compute the GW merger time by calculating the orbital evolution in full as outlined by Peters (1964) rather than by simple interpolation between the two analytic forms for circular and highly eccentric orbits. This is motivated by the fact that many of the orbits lie in the mid-range between circular and eccentric after the second supernova, and the updated calculations offer a more accurate prediction of the merger times.

\subsection{Detection horizons}

Detection of predicted mergers will depend on instrumental and alignment effects. Suites of codes to evaluate this do exist, such as LALSuite (LIGO Scientific Collaboration 2018), but we implement our own Python package to do this because of our specific focus on population synthesis. The package RIRORIRo ${ }^{2}$ has been created with the aim of determining the fraction of predicted GW transients that can be detected (van Zeist et al. 2021). Currently, it only deals with GW transients caused by the merger of NSNS, NS$\mathrm{BH}$, and $\mathrm{BHBH}$. In the future, we plan to extend its scope to all areas of the GW population and the resulting GW spectral synthesis.

For each chirp mass and mass ratio pair RIRORIRO creates a synthetic merger gravitational waveform (including inspiral and ringdown phases). The effects of source orientation and distance are then taken into account to calculate an effective detection volume and the evolution of detection fraction of these events with luminosity distance. Our calculations span from $1 \mathrm{Mpc}$ to $10 \mathrm{Gpc}$ in steps of 0.1 dex. The package is inspired from Buskirk \& Babiuc-Hamilton (2019) who created a Mathematica routine to calculate the expected GW waveform for a GW transient event. They used the results of Huerta et al. (2017) to model the inspiral and ringdown emission. We use the same methods they outline as well as using information from the FindCHIRP algorithm (Allen et al. 2012) to improve our modeling of the inspiral waveform and calculation of the signal-to-noise ratio (SNR). It is often difficult to match the inspiral and ringdown waveforms and we switch between them when the frequency and time derivative are closest between the two models.

For each resultant waveform, we use the method of Barrett et al. (2018) to calculate the SNR of the merger model at a given redshift using the corresponding luminosity distance and redshifting the waveform. We subsequently calculate a total SNR assuming a triple-detection by combining the SNR calculated for the LIGO Hanford, LIGO Livingston, and VIRGO detectors during O3 in quadrature. We also calculate the average expected SNR of a GW observation with a single, non-specific detector operating. These SNR values assumed optimal alignment of detector and source. To

2 A riroriro is a bird native to Aotearoa New Zealand that is frequently heard but seldom seen. 
take into account the effect of arbitrary orientation we multiplied these with a projection function (Finn 1996; Belczynski et al. 2013, 2014), giving a probability distribution of the expected SNR and the probability that this would exceed the commonly-used detectability threshold value of 8 , i.e. the detection probability. The variation in the detection probability of a GW transient depending on the chirp mass, mass ratio, and distance of the binary is illustrated in Fig. 1. For each chirp mass, there is a maximum distance beyond which no mergers can be detected but there is a smoothly decreasing detection probability up to this horizon. Higher chirp masses can be detected to larger distances. However, at the highest chirp masses a hard upper limit is introduced due to the decreasing maximum frequency of these mergers. This upper limit is most sensitive to the mass ratio, as shown in Fig. 1, and the lowest mass ratio has a significantly lower maximum chirp mass for the peak comoving distance to the source. We combine the detection probabilities as a function of chirp mass, mass ratio and distance with the population statistics from BPASs, which dictate the frequency with which systems of such parameters form, to create a forward model for the LVC detection rates. We integrate the rates in events per year per unit volume over the observable Universe to obtain rates in events per year. We use the method of Hogg (1999) to convert the luminosity distances used in the previous steps to comoving distances which are employed in the volume calculations ${ }^{3}$. We reduce our rates by a factor of (177.3 +142.0 ) days / 365.25 days) to account for the elapsed duration during $\mathrm{O} 3 \mathrm{a}+\mathrm{O} 3 \mathrm{~b}$ where at least one detector was active and taking data.

\subsection{Remnant mass estimates and supernova kick velocities}

Within BPASs the standard method of calculating remnant masses at the end of stellar evolution for massive stars is to compare the binding energy of the stellar envelope to the typical SN explosion energy of $10^{51}$ ergs. The ejecta mass is considered to be the amount of mass with this much binding energy, removed from the star, and the remainder goes into the remnant mass (Eldridge \& Tout 2004). Here we refer to this method as the Standard remnant mass scheme. However, to explore the effect of varying the remnant mass prescription on our predictions, we look into three other schemes.

(i) In AlwaysNS scheme, motivated by the Chandrasekhar mass limit (and to save computation time), we force all the models to form a neutron star (NS) of $1.4 \mathrm{M}_{\odot}$ at core-collapse. Forcing all the remnants to form a NS at birth (albeit of the same mass) allows us to observe the influence this could have on the NS mergers rates. (ii) For most stars the final mass of the carbon-oxygen (CO) core would be a fair estimate of the maximum possible remnant mass, hence in $\mathrm{M}_{\mathrm{CO} \text {,final }}$ remnant mass scheme, the final $\mathrm{CO}$ core mass of the progenitor model is set equal to the remnant mass (i.e. only the hydrogen/helium envelope will be ejected, independent of SN energy). (iii) For comparative purpose we employ a commonlyused rapid synthesis scheme from Fryer et al. (2012), here referred as FryerRapid. This scheme gives a remnant mass that depends on

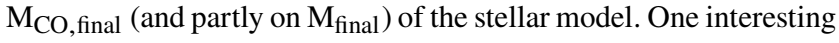
feature is that it predicts a mass-gap in the $\mathrm{BH}$ mass distribution with no BHs less massive than $5 \mathrm{M}_{\odot}$. Consequently, using the FryerRapid scheme allows us to determine the impact of allowing for a mass-gap. Finally, in addition to using the above remnant mass prescriptions, we also use two different SN kick schemes namely the Hobbs et al. (2005) and Bray \& Eldridge (2018) kick

3 We assume a standard $\Lambda \mathrm{CDM}$ cosmology with $H_{0}=100 \mathrm{hms}^{-1} \mathrm{Mpc}^{-3}$ with $\mathrm{h}=0.696, \Omega_{\mathrm{M}}=0.286, \Omega_{\Lambda}=0.714$
Table 1. Predicted number of observable detections during $\mathrm{O} 3(\mathrm{a}+\mathrm{b})$ for the three expected mergers types, employing various methods for calculating SN kicks and remnant masses. Rates in parenthesis are local intrinsic merger rates (in units of events $\mathrm{Gpc}^{-3} \mathrm{yr}^{-1}$ ) calculated before the consideration of detection probabilities. Last row states the LVC O3 $(a+b)$ observed and intrinsic rates. (Abbott et al. 2020a; The LIGO Scientific Collaboration et al. 2021b). The detectable rates account for the duration of $177.3+142.0$ days of $\mathrm{LVC} \mathrm{O} 3$ during which at least one detector was active.

\begin{tabular}{ccccc}
\hline Kick & Remnant & NSNS & BHNS & BHBH \\
\hline Hobbs & Standard & $4.9(208)$ & $12.6(131)$ & $45(31)$ \\
Hobbs & M $_{\text {CO, final }}$ & $1.6(43)$ & $46.8(417)$ & $420(873)$ \\
Hobbs & FryerRapid & $3.8(176)$ & $27(208)$ & $171(169)$ \\
Hobbs & AlwaysNS & $4.9(223)$ & $0.4(8.7)$ & 0.0 \\
\hline Bray & Standard & $17.1(745)$ & $13.3(180)$ & $27.0(13)$ \\
Bray & M $_{\text {CO, final }}$ & $5.6(179)$ & $43.2(498)$ & $212.5(569)$ \\
Bray & FryerRapid & $13.7(677)$ & $16.0(157)$ & $64.8(70)$ \\
Bray & AlwaysNS & $14.4(708)$ & $2(48)$ & 0.0 \\
\hline \hline LVC O3(a+b) & $1 \pm 1$ & $5 \pm 2.2$ & $69 \pm 8.3$ \\
Intrinsic $\left[\mathbf{G p c}{ }^{-3} \mathbf{y r}^{-1}\right.$ ] & $(13-1900)$ & $(7.4-320)$ & $(17.3-45)$ \\
\hline
\end{tabular}

velocity relations. The former is described by a Maxwell-Boltzmann distribution with 1-dimensional root mean square speed of 265 $\mathrm{kms}^{-1}$ while the latter depends strongly by construction on the masses of the ejecta and the remnant and thus we expect the kick and remnant mass distributions to interact in a complex and nonlinear way. Mathematically, the Bray kick relation is as follows

$v_{\text {kick }}=100\left(\frac{M_{\text {ejecta }}}{M_{\text {remnant }}}\right)-170\left(\frac{1.4}{M_{\text {remnant }}}\right)$

where $v_{\text {kick }}$ is the velocity, in $\mathrm{km} \mathrm{s}^{-1}$, imparted to the compact remnant as a result of the $\mathrm{SN}, M_{\text {ejecta }}$ is the $\mathrm{SN}$ ejecta mass and $M_{\text {remnant }}$ is the remnant mass (all masses in $\left[\mathrm{M}_{\odot}\right]$ units).

\section{RESULTS}

Below we show some ways in which one could constrain or verify the form of the SNe kick and the remnant mass relation using the $\mathrm{GW}$ transient data from $\mathrm{O} 3$.

\subsection{Some implications based on merger rates}

Our results shown in Table 1 (also see Fig. A1, A2 in the Appendix), presents the expected number of events that should have been detected in $\mathrm{O} 3$ along with the predicted local intrinsic rates under each set of prescriptions. On considering these under the light of $\mathrm{O} 3$ merger rate data, we observe patterns with interesting implications:

(i) The Bray kick consistently gives a low BHBH intrinsic and detectable merger rate, as compared to the Hobbs kick. The strength of these differences varies between the remnant mass schemes but a general trend holds. This is acceptable as in the Bray kick, $\mathrm{v}_{\mathrm{kick}}$ is inversely proportional to the $\mathrm{M}_{\text {remnant }}$. Thus more massive remnants receive weaker kicks and so have less eccentric orbits, longer merger times and a lower BHBH merger rate. In particular, we find that for very massive $\mathrm{BHs}$ the eccentricity of the orbit is independent of the kick but for intermediate mass BHs, Hobbs kick produces more eccentric orbits and therefore more mergers. This behavior can be seen in Fig. 2 where the cumulative observable count for both kicks closely follow each other up till $\sim 1.35\left(\sim 22 \mathrm{M}_{\odot}\right)$ from the left, after which Hobbs kick starts to produce more observable detection for the BBH systems. 


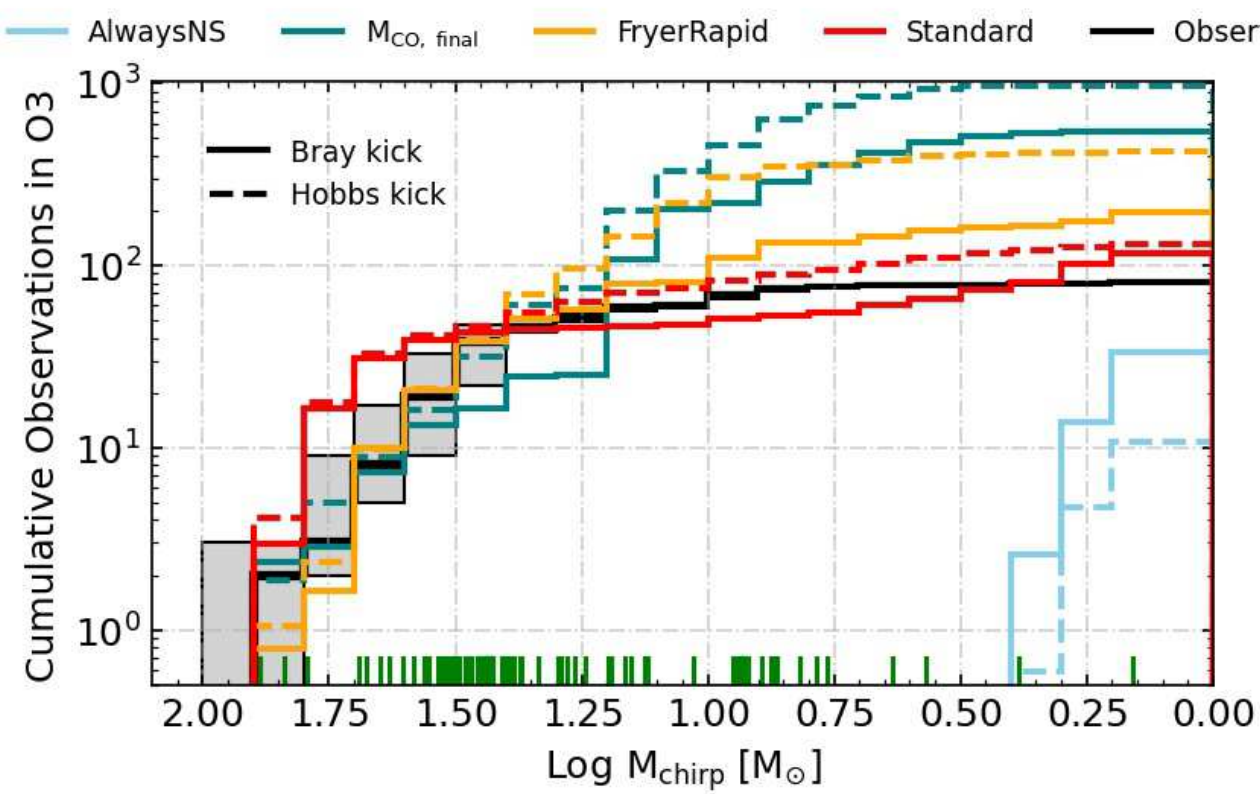

Figure 2. The Chirp mass distributions of cumulative events observed by LVC O3(a+b) (black line) and those predicted by various schemes under the BPASS model populations for the same duration of observation. The green ticks show the observed values of $\mathrm{M}_{\text {chirp }}$ and the solid grey area represents the uncertainty between the upper and lower $\mathrm{M}_{\text {chirp }}$ bounds for these observed events (Abbott et al. 2020a; The LIGO Scientific Collaboration et al. 2021b). The black curve is the cumulative observed number of events over the observed values of $\mathbf{M}_{\text {chirp }}$. Solid lines represent Bray kick while dashed lines Hobbs kick.

(ii) In $\mathrm{M}_{\mathrm{CO} \text {,final }}$, the $\mathrm{BHBH}$ and BHNS intrinsic and detected rates are significantly higher than those seen in $\mathrm{O} 3$ implying that under our current settings, stellar remnants must be consistently less massive than the $\mathrm{M}_{\mathrm{CO}}$, final of their progenitors. Fig. A6 (discussed later) also demonstrates this bias of $\mathrm{M}_{\mathrm{CO}}$,final toward higher mass remnants.

(iii) The Standard scheme over-predicts the number of detectable NSNS and BHNS mergers while under-predicting the BHBH mergers (fig A1), more so using the Bray kick. On the other hand, a better agreement is seen once their intrinsic merger rates are compared with the expected local intrinsic rate. Additionally, the $\mathrm{O} 3 \mathrm{BHBH}$ merger count is reasonably reproduced by the Bray kick + FryerRapid remnant scheme. However, this produces too many mergers involving binary NSs. Though none of the schemes fully satisfy either the detectability or intrinsic criteria set by LVC O3, the Standard + Hobbs and Fryer Rapid + Bray offer the closest fit to the data in Table 1. This can also be seen in figure A3 which provides the local BHBH merger rate density distribution as a function of the primary BH's mass. Both the Standard + Hobbs and Fryer Rapid + Bray produce mergers owing only to the isolated binary evolution scenario and their rates mostly fall below the $90 \%$ credibility interval. Other relations also show similar trends but the above two schemes along with Fryer Rapid + Hobbs come the closest to the PowerLaw+Peak distribution. We note that though Fig. A3 helps us to perform a check on the validity of the chosen schemes it is not yet possible to reach a definitive conclusion.

(iv) The AlwaysNS scheme does have some BHNS mergers despite no BHs being formed in core-collapse. These BHs are the result of the subsequent accretion-induced collapse (due to accretion from the companion) of the already formed NS. This gives an indication of the relative importance of such a channel compared to normal BHNS mergers where one of the BHs is formed directly at core-collapse.

(v) On comparing Fig. A1 and A2 we find that most NSNS and BHNS rates fail to fall within the allowed detectability range.
On the other hand, a better agreement is seen once their intrinsic merger rates are compared with the expected local intrinsic rates (Fig. A2). Also, we find that there are some variations in the relative magnitudes of the intrinsic rates in comparison to the detectable rates for the same underlying remnant mass and SNe kick scheme. At present, we think that more detections (especially of NSNS and BHNS mergers) need to be performed before any substantive conclusion can be drawn from these trends.

\subsection{Implications based on the chirp mass distribution}

Using rates alone is a limited approach to constrain the models. We must consider the number of events expected in chirp mass and mass ratio space as well. To this end, we first consider the predicted chirp mass distribution of our model GW transient populations. Fig. 2 shows the cumulative number of GW transients versus chirp mass from the high-mass end of the chirp mass distribution. We see that most of the model predictions reasonably match the observations at the high-mass end down to $\log 1.4$ (i.e. $\sim 25 \mathrm{M}_{\odot}$ ), although the Standard scheme (both kicks) tends to predict a higher count of massive mergers than observed. This can be seen more clearly in Fig. A4 which provides the differential numbers for the same. Below $\sim 25 \mathrm{M}_{\odot}$, the predicted distributions diverge significantly, with most schemes predicting more GW transients of lower chirp mass than observed. In the mass range of $\log 1.4-\log 0.5\left(\sim 25-5 M_{\odot}\right)$ Hobbs kick produces more observable mergers but at the lowest chirp masses (spanning BHNS/NSNS mergers), populations arising from Bray kick have an excess of systems. Cumulatively, the Hobbs kick tends to produce more detectable systems. However, among the schemes presented here, Fig. 2 and A4 makes it possible to identify those that offer the closest match for the observed cumulative and differential events count over the $\mathrm{M}_{\text {chirp }}$ range. 

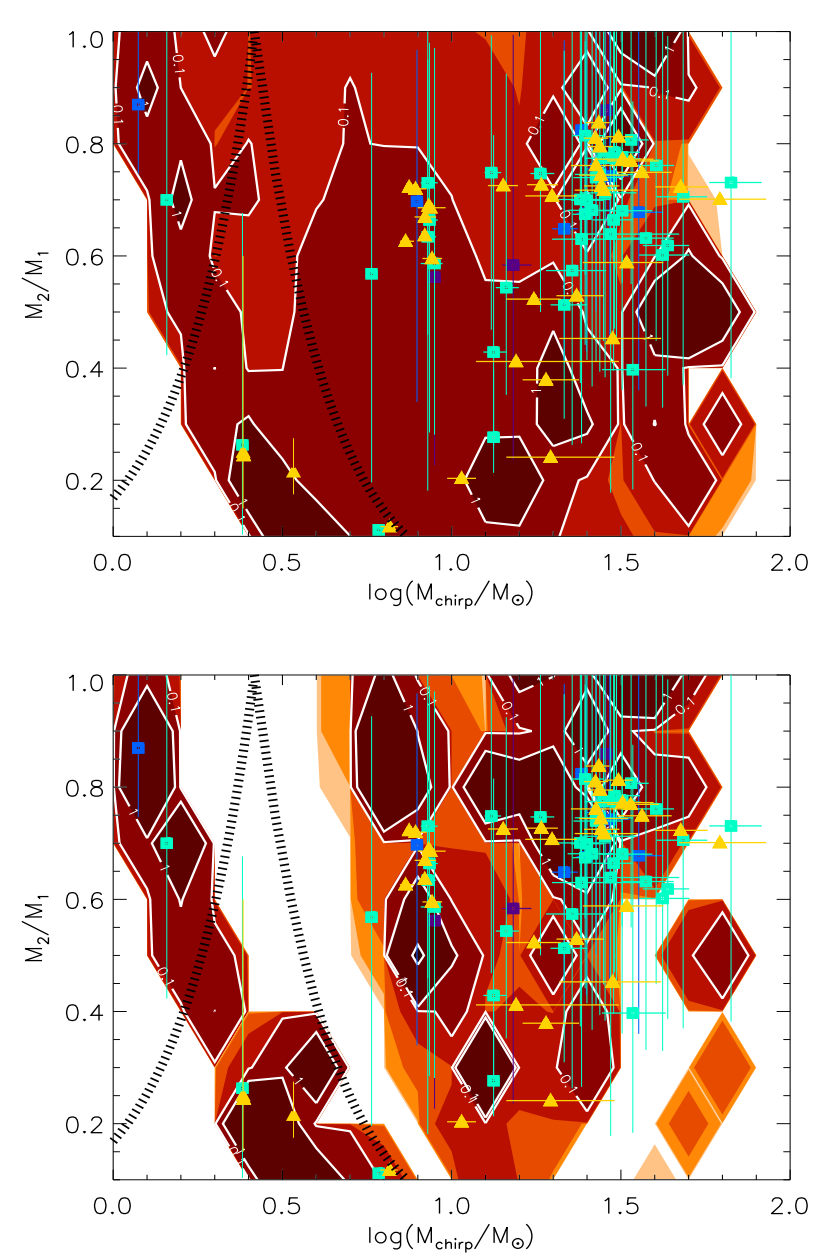

Figure 3. Contour plots of the expected distributions of detected events in $\mathrm{O} 3$ in $\mathrm{M}_{\text {chirp }}$ and mass ratio space. The upper panel shows the Hobbs kick and the Standard remnant mass scheme, the lower panel shows the Bray kick and the FryerRapid remnant mass scheme. Darker contours represent a higher probability. The black dotted line distinguishes the regions where mergers occur with component masses both either below $3 \mathrm{M}_{\odot}$, one above and one below or both above $3 \mathrm{M}_{\odot}$ (i.e, the expected regions of NSNS, BHNS, and BHBH mergers). The dark blue symbols are the observed events from O1, the blue symbols are from $\mathrm{O} 2$, the cyan symbols from O3a and the yellow triangles from $\mathrm{O} 3 \mathrm{~b}$.

\subsection{Implications based on chirp mass - mass ratio distribution}

We now further the analysis by comparing the GW transient distributions in chirp mass and mass ratio $\left(M_{\text {chirp }}\right.$ vs $\left.q\right)$ space. Fig. 3, shows the schemes that offer the closest fit to the total event rates in Table 1 (i.e., Standard + Hobbs kick and FryerRapid + Bray kick). While the Standard scheme spans nearly all combinations of remnant mass, however, in the FryerRapid scheme the mass gap (Bailyn et al. 1998; Özel et al. 2010) becomes apparent due to a clear lack of BH systems near the BHNS/BHBH dividing lines.

For $\log \left(\mathrm{M}_{\text {chirp }} / \mathrm{M}_{\odot}\right)$ in the neighborhood of $\log 0.7$ (i.e. $5 \mathrm{M}_{\odot}$ ) - close to the mass-gap end, - the Standard scheme overpredicts the number of expected mergers over most parts of the $q$ interval. The FryerRapid scheme in Fig. 3 does achieve a reasonably accurate peak near $M_{\text {chirp }} \sim 1.5$ and $q \sim 0.7$. However, it predicts a second, much larger peak for $M_{\text {chirp }}$ and $q \sim 0.8$ and a stronger
BHNS peak. In Fig. 3 the peaks in the predicted and observed number of events for larger $M_{\text {chirp }}$ lie at similar but not identical $M_{\text {chirp }}$ vs $q$ ranges. This suggests a need for further investigation at the upper end of the $\mathrm{BH}$ mass distribution, close to the mass range for pair-instability SNe (e.g. Woosley 2017; Stevenson et al. 2019). Additionally, in Fig. A5, the Standard + Bray kick scheme underpredicts the BHBH and over-predicts the NSNS events, consistent with the findings in fig 2, A1 and A4. The FryerRapid + Hobbs kick scheme in Fig. A5 also fails to provide a reliable match for the total events over the $M_{\text {chirp }}$ vs $q$ range. The $M_{\mathrm{CO}}$, final scheme in Fig. A6 produces too many mergers nearly over the entire $q$ range at the lower and intermediate $\mathbf{M}_{\text {chirp }}$ values. In our simulation, these mergers could be avoided if the remnant mass is consistently less than $M_{\mathrm{CO}}$, final in this $M_{\text {chirp }}$ range which leads to a stronger kick and wider orbits. Overall, on comparing the distribution of the observed GW transients to our forward-model predictions, we find that the small number statistics still limit a meaningful comparison.

\section{DISCUSSION \& CONCLUSIONS}

Here, we have presented theoretical predictions of the expected number of events (and their distribution in chirp mass and mass ratio space) detected within BPASS under the LVC O3 sensitivity criteria. We find that none of the remnant mass schemes and SN kick combinations presented fully describe the observed population of GW transients in the $\mathrm{O} 3$ catalogs. Although most population models do reasonably well in reproducing the chirp mass distribution at masses above $\sim 25 \mathrm{M}_{\odot}$ but diverge considerably at lower masses. Our results suggest that the Standard + Hobbs kick scheme performs the best overall but requires modification at the upper and lower ends of the chirp mass distribution. The values of the most massive chirp masses predicted by this scheme need to be reduced by 0.1-0.2 dex, while the mass-gap needs to be included in some form at the lower end of the $\mathrm{BH}$ mass distribution. However, since the event GW190814 (Abbott et al. 2020b) is not predicted in the FryerRapid scheme, this suggests that a prescription making low mass $\mathrm{BHs}$ significantly rarer, rather than preventing them from forming entirely within the mass-gap, may be required (see also, Zevin et al. 2020). The Standard + Hobbs kick somewhat underpredicts the observed BHBH events in Table 1, (though it satisfies the intrinsic merger rate constraint) but these are the outcome of isolated binary evolution only (e.g. see, Zevin et al. 2021). We note that here we only vary the SNe remnant mass and SNe kick schemes while keeping the other uncertain parameters fixed (see, Eldridge et al. 2017). Hence the predicted values have an implicit uncertainty built into them. Moreover, our models do not yet allow for tidally-induced chemical homogeneous evolution which (see de Mink et al. 2009; de Mink \& Mandel 2016; Marchant et al. 2016) could result in a higher BHBH merger rate. Additionally, BHBH mergers could also come from dynamical channels in young stellar or globular clusters (e.g., Kulkarni et al. 1993; Portegies Zwart \& McMillan 2000) or at the disks of active galactic nuclei (e.g., McKernan et al. 2012) and some BHBH systems could be of primordial origin, arising in the early Universe (Carr \& Hawking 1974). Tang et al. (2020) found that the GW transient rates involving BHs are highly dependent on the rate of chemical enrichment of the Universe and the number of BHNS and BHBH mergers can be reduced by altering the cosmic metallicity evolution. However, rapid enrichment would be required for our highest predicted rates to match those observed in O3. Lastly, all the predictions made here, assume fixed values for the rest of the uncertain parameters that go into the binary stellar 
evolution (see Eldridge et al. 2017 for more information). Changing these parameters could affect the results of this paper and hence any reliable prediction can only be made when more constraints (for the other unknowns) are available from other independent sources.

To conclude, we find some of our model populations produce a reasonable agreement between the predictions and observed distributions. Nonetheless, discrepancies remain, highlighting remaining uncertainties in our understanding of high mass compact binary formation. Our results indicate that no one model completely matches the observed distribution, although the Standard remnant mass scheme + Hobbs kick and FryerRapid scheme + Bray kick offer the closest match. The key features which are difficult to match for these model sets are the smaller number of low chirp mass transients and a significant peak around $\log 1.48\left(\sim 30 \mathrm{M}_{\odot}\right)$. In the future, we might consider using a prescription where both the kick and remnant mass are stochastically sampled, similar to Mandel \& Müller (2020). Additionally, at the high mass end the physics of pair-instability and pulsational pair-instability systems also need to be taken into account (as in Stevenson et al. 2019). In Fig. A1 and A2, we find variations in the relative magnitudes of the intrinsic rates in comparison to the detectable rates for the same underlying remnant mass and SNe kick scheme. Given that supernovae remnant masses and kicks are interrelated, higher number statistics are needed to fully explore their effects. This should be possible as GW transient catalogs grow in size and completeness.

\section{ACKNOWLEDGEMENTS}

We thank the anonymous referee for their helpful feedback and suggestions. SG and WGJvZ acknowledge support from the University of Auckland. JJE and HFS acknowledge the support of the Marsden Fund Council managed through the Royal Society of New Zealand Te Apārangi. ERS received support from United Kingdom Science and Technology Facilities Council (STFC) grant ST/T000406/1.

\section{DATA AVAILABILITY}

The data underlying this article will be shared on reasonable request to the corresponding author.

\section{REFERENCES}

Abbott B. P., et al., 2016, Phys. Rev. Lett., 116, 241102

Abbott B. P., et al., 2017, Phys. Rev. Lett., 119, 161101

Abbott B., et al., 2019, Physical Review X, 9, 031040

Abbott R., et al., 2020a, arXiv e-prints, p. arXiv:2010.14527

Abbott R., et al., 2020b, ApJ, 896, L44

Allen B., Anderson W. G., Brady P. R., Brown D. A., Creighton J. D. E., 2012, Phys. Rev. D, 85, 122006

Bailyn C. D., Jain R. K., Coppi P., Orosz J. A., 1998, ApJ, 499, 367

Barrett J. W., Gaebel S. M., Neijssel C. J., Vigna-Gómez A., Stevenson S., Berry C. P. L., Farr W. M., Mandel I., 2018, MNRAS, 477, 4685

Belczynski K., Kalogera V., Rasio F. A., Taam R. E., Zezas A., Bulik T., Maccarone T. J., Ivanova N., 2008, ApJS, 174, 223

Belczynski K., Bulik T., Mandel I., Sathyaprakash B. S., Zdziarski A. A., Mikołajewska J., 2013, ApJ, 764, 96

Belczynski K., Buonanno A., Cantiello M., Fryer C. L., Holz D. E., Mandel I., Miller M. C., Walczak M., 2014, ApJ, 789, 120

Belczynski K., Holz D. E., Bulik T., O’Shaughnessy R., 2016, Nature, 534, 512

Boco L., Lapi A., Chruslinska M., Donevski D., Sicilia A., Danese L., 2021, ApJ, 907, 110

Bray J. C., Eldridge J. J., 2018, MNRAS, 480, 5657

Broekgaarden F. S., et al., 2021, arXiv e-prints, p. arXiv:2103.02608

Buskirk D., Babiuc-Hamilton M. C., 2019, EJP, 40, 025603

Carr B. J., Hawking S. W., 1974, MNRAS, 168, 399

Chruslinska M., Nelemans G., Belczynski K., 2019, MNRAS, 482, 5012
De Marco O., Izzard R. G., 2017, Publ. Astron. Soc. Australia, 34, e001

Eldridge J. J., Stanway E. R., 2016, MNRAS, 462, 3302

Eldridge J. J., Tout C. A., 2004, MNRAS, 353, 87

Eldridge J. J., Stanway E. R., Xiao L., McClelland L. A. S., Taylor G., Ng M., Greis S. M. L., Bray J. C., 2017, PASA, 34, e058

Eldridge J. J., Stanway E. R., Tang P. N., 2019, MNRAS, 482, 870

Finn L. S., 1996, Phys. Rev. D, 53, 2878

Fishbach M., Kalogera V., 2021, arXiv e-prints, p. arXiv:2105.06491

Fryer C. L., Belczynski K., Wiktorowicz G., Dominik M., Kalogera V., Holz D. E., 2012, ApJ, 749, 91

Giacobbo N., Mapelli M., Spera M., 2018, MNRAS, 474, 2959

Hobbs G., Lorimer D. R., Lyne A. G., Kramer M., 2005, MNRAS, 360, 974

Hogg D. W., 1999, arXiv e-prints, pp astro-ph/9905116

Huerta E. A., et al., 2017, Phys. Rev. D, 95, 024038

Hurley J. R., Pols O. R., Tout C. A., 2000, MNRAS, 315, 543

Ivanova N., et al., 2013, A\&ARv, 21, 59

Kulkarni S. R., Hut P., McMillan S., 1993, Nature, 364, 421

LIGO Scientific Collaboration 2018, LIGO Algorithm Library - LALSuite, Free software (GPL), doi:10.7935/GT1W-FZ16

Lipunov V., Gorbovskoy E., Grinshpun V., Vlasenko D., 2021, MNRAS, 502, 1925

Maeder A., 1987, A\&A, 178, 159

Mandel I., Müller B., 2020, MNRAS, 499, 3214

Mapelli M., Giacobbo N., Ripamonti E., Spera M., 2017, MNRAS, 472, 2422

Marchant P., Langer N., Podsiadlowski P., Tauris T. M., Moriya T. J., 2016, A\&A, 588, A50

McKernan B., Ford K. E. S., Lyra W., Perets H. B., 2012, MNRAS, 425, 460

Özel F., Psaltis D., Narayan R., McClintock J. E., 2010, ApJ, 725, 1918

Patton R. A., Sukhbold T., 2020, MNRAS, 499, 2803

Peters P. C., 1964, Physical Review, 136, 1224

Portegies Zwart S. F., McMillan S. L. W., 2000, ApJ, 528, L17

Stanway E. R., Eldridge J. J., 2018, MNRAS, 479, 75

Stevenson S., Vigna-Gómez A., Mandel I., Barrett J. W., Neijssel C. J., Perkins D., de Mink S. E., 2017, Nature Communications, 8, 14906

Stevenson S., Sampson M., Powell J., Vigna-Gómez A., Neijssel C. J., Szécsi D., Mandel I., 2019, ApJ, 882, 121

Tang P. N., Eldridge J. J., Stanway E. R., Bray J. C., 2020, MNRAS, 493, L6

The LIGO Scientific Collaboration the Virgo Collaboration the KAGRA Collaborationn 2021a, doi:10.5281/zenodo.5655785, https://doi.org/10.5281/zenodo. 5655785

The LIGO Scientific Collaboration et al., 2021b, arXiv e-prints, p. arXiv:2111.03606

Vigna-Gómez A., et al., 2018, MNRAS, 481, 4009

Woosley S. E., 2017, ApJ, 836, 244

Woosley S. E., Blinnikov S., Heger A., 2007, Nature, 450, 390

Zevin M., Spera M., Berry C. P. L., Kalogera V., 2020, ApJ, 899, L1

Zevin M., et al., 2021, ApJ, 910, 152

de Mink S. E., Mandel I., 2016, MNRAS, 460, 3545

de Mink S. E., Cantiello M., Langer N., Pols O. R., Brott I., Yoon S. C., 2009, A\&A, 497, 243

van Zeist W. G. J., Stevance H. F., Eldridge J. J., 2021, The Journal of Open Source Software, 6, 2968

\section{APPENDIX A: SUPPLEMENTARY FIGURES FOR GW EVENT DISTRIBUTION}

This appendix presents a visual plot (Fig. A1, A2) of the data shown in Table 1, the local merger rate density distribution as a function of the primary BH's mass (Fig. A3) for the various schemes, the differential rates (Fig. A4) for data shown in Fig. 2 and also the chirp mass - mass ratio distributions associated with the additional supernova kick and remnant mass prescriptions as discussed in the text (Fig. A5, A6).

This paper has been typeset from a $\mathrm{T}_{\mathrm{E}} \mathrm{X} / \mathrm{L} \mathrm{AT} \mathrm{E} \mathrm{X}$ file prepared by the author. 


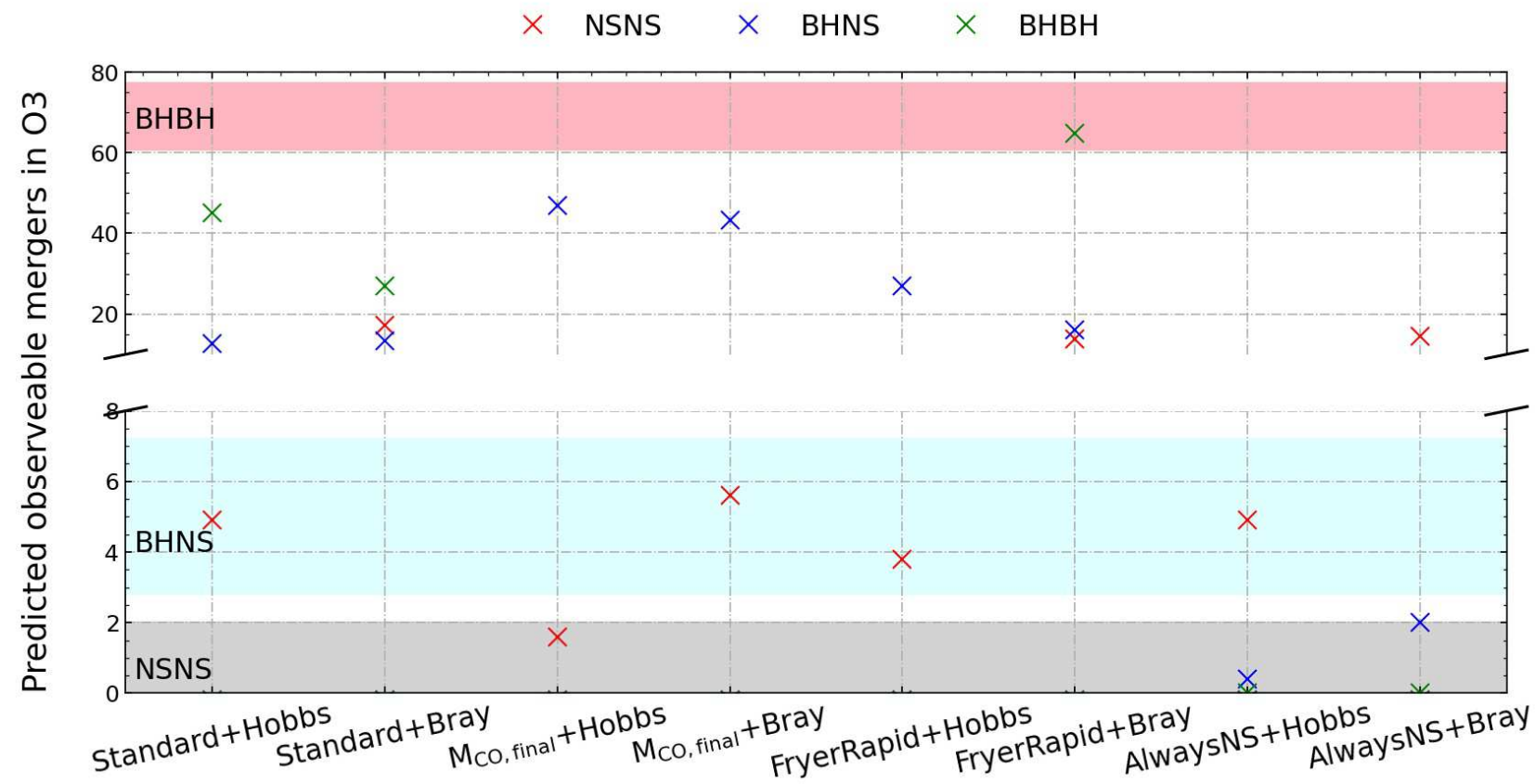

Figure A1. To assist in reading, here we plot the predicted number of detectable mergers in $\mathrm{O} 3$ as shown in Table 1 by the various schemes. Pink band represents the range of allowed values for BHBH, cyan for BHNS and grey for NSNS merger rates as obtained by LVC O3, (Abbott et al. 2020a; The LIGO Scientific Collaboration et al. 2021b). The red crosses are for NSNS, blue for BHNS and green for BHBH merger rates. The BHBH merger rate for

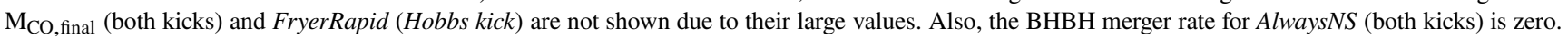

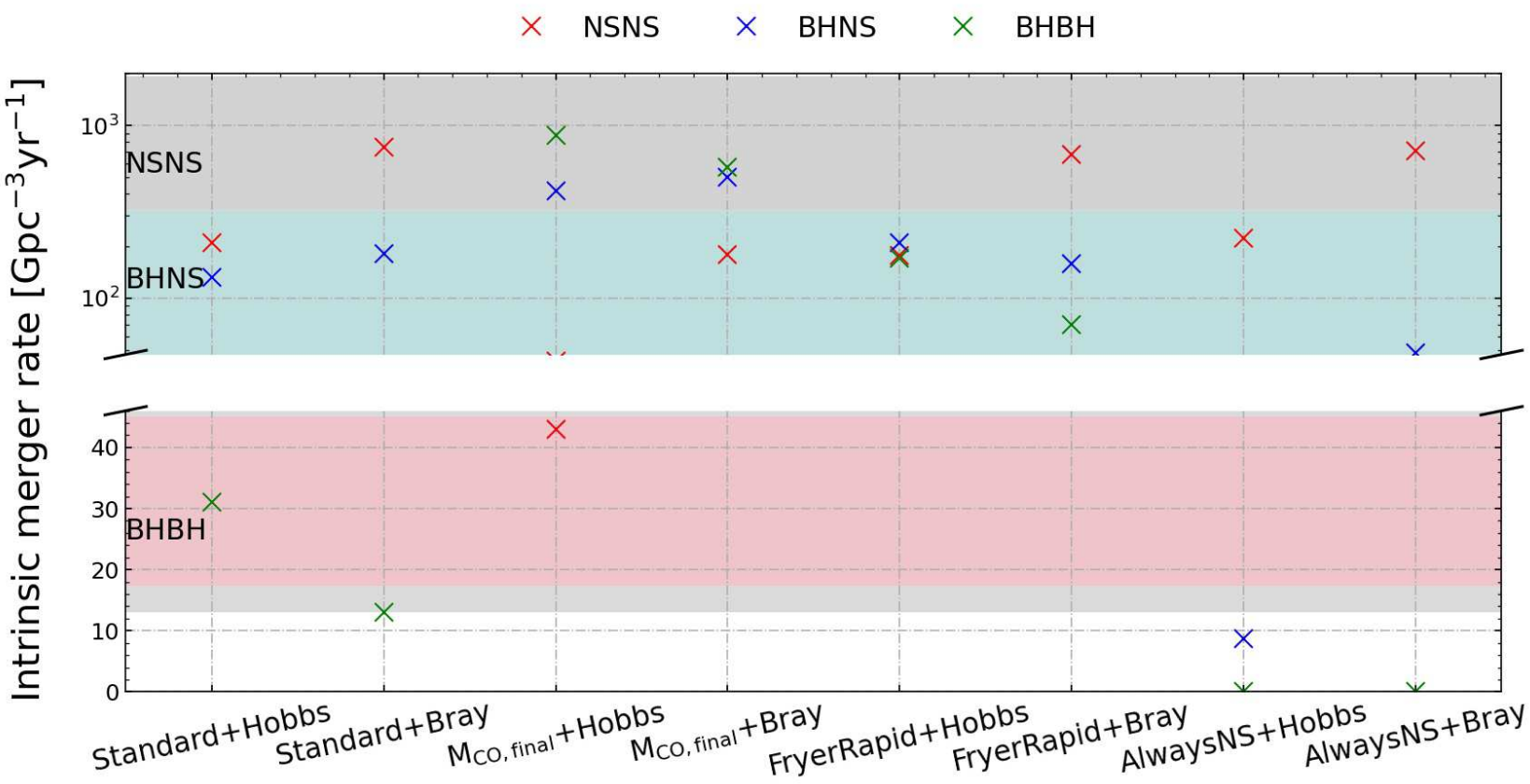

Figure A2. Here we plot the intrinsic local merger rates in $\mathrm{Gpc}^{-3} \mathrm{yr}^{-1}$ as shown in Table 1 by the various schemes. Pink band represents the range of allowed values for BHBH, cyan for BHNS and grey for NSNS merger rates as obtained by LVC O3, (Abbott et al. 2020a; The LIGO Scientific Collaboration et al. 2021b) using their assumptions for the underlying remnant mass distribution (which differs from those explored here). The overlapping cyan and grey region represents the overlap in the allowed ranges for the NSNS and BHNS mergers rates. The crosses have the same meaning. The BHBH merger rate for Always NS (both kicks) is zero. Note the change of scale on y-axis. 


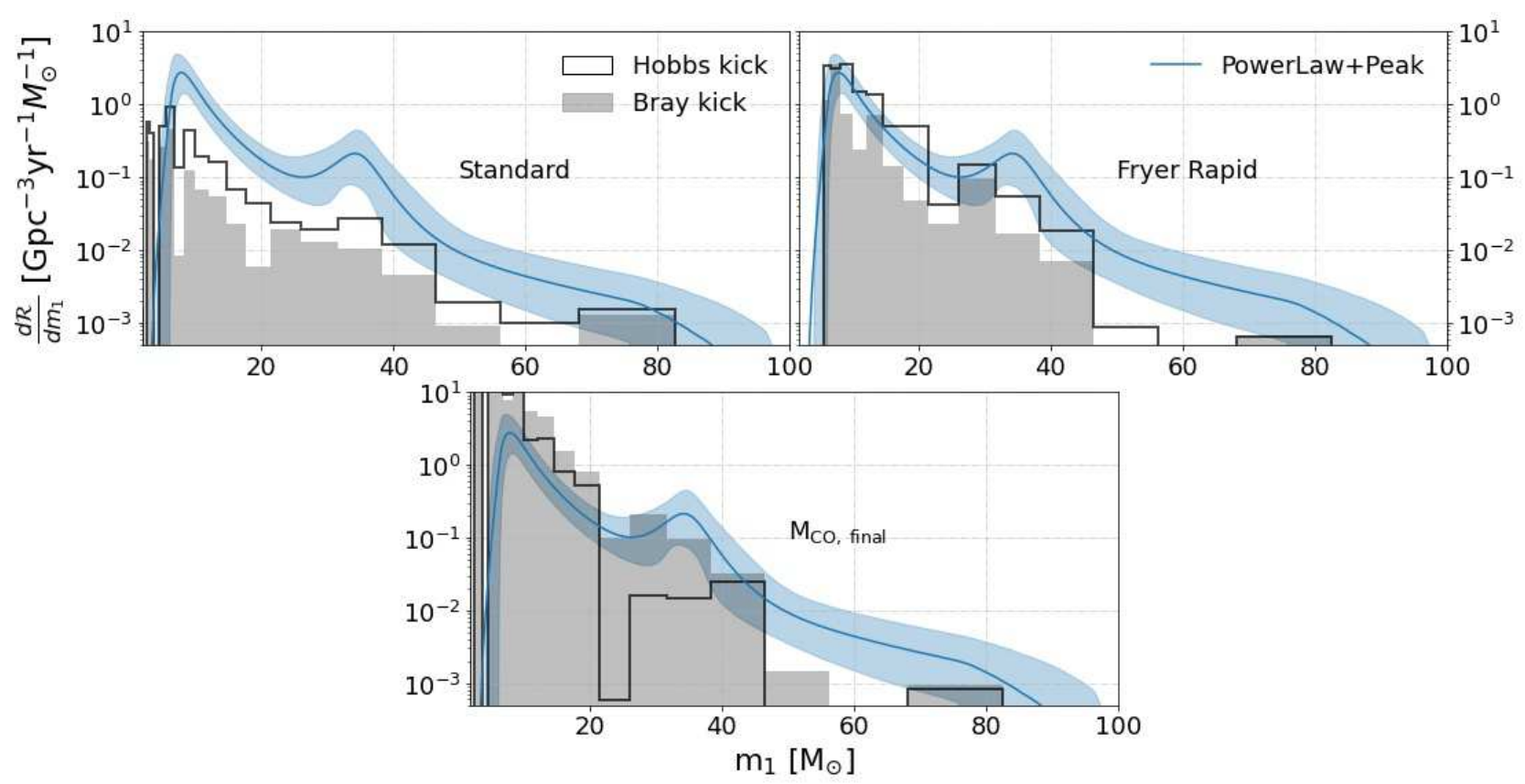

Figure A3. The local merger rate density (per unit solar mass) distribution as a function of the primary BH's mass in the BBH mergers for the various schemes. The blue curve is the PowerLaw+Peak posterior population distribution for the same with its $90 \%$ credible interval, represented by the shaded extension (the PowerLaw+Peak curve has been reproduced from The LIGO Scientific Collaboration et al. 2021a which is based on the work of The LIGO Scientific Collaboration et al. 2021b). The slope $(\alpha)$ of the powerlaw in $\log -\log$ space is $\alpha=3.4_{-0.49}^{+0.58}$ supplemented with a Gaussian peak at $34_{-3.8}^{+2.3} M_{\odot}$. The unshaded histogram is for Hobbs while the shaded is for Bray kick respectively. The bins are logarithmically spaced and the minimum birth mass of $\mathrm{BH}$ is set to $2.5 \mathrm{M}_{\odot}$ resulting in systems on the LHS of $3 \mathrm{M}_{\odot}$ as well. The presence of systems in the pair-instability region is due to subsequent accretion by the BH from the companion star. Moreover, the data presented here does not yet account for the pulsational pair-instability SNe (e.g. Woosley et al. 2007) and therefore does not feature a clear peak around $34 \mathrm{M}_{\odot}$.

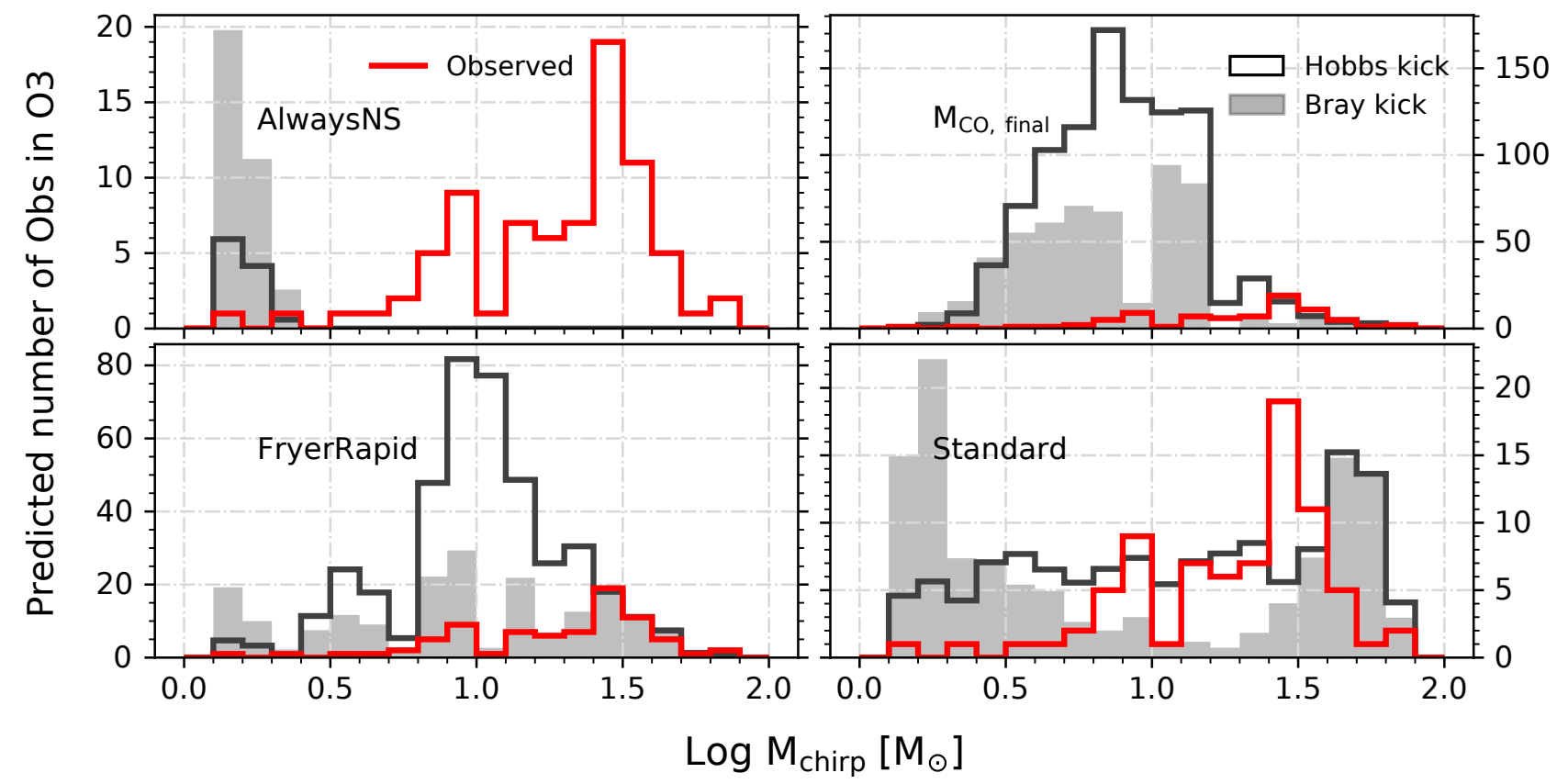

Figure A4. The predicted number of observations by various schemes as a function of chirp mass. The red histogram represents the number of observed events in $\mathrm{O} 3$ in their respective mass bin. The filled histogram represent Bray kick while the empty ones represent Hobbs kick. Note that the y-scale varies among the plots. Apart from the Standard + Hobbs and FryerRapid + Bray, others schemes predict large (or tiny) peaks at various $\mathrm{M}_{\text {chirp }}$ values that are highly inconsistent with the observed data. 

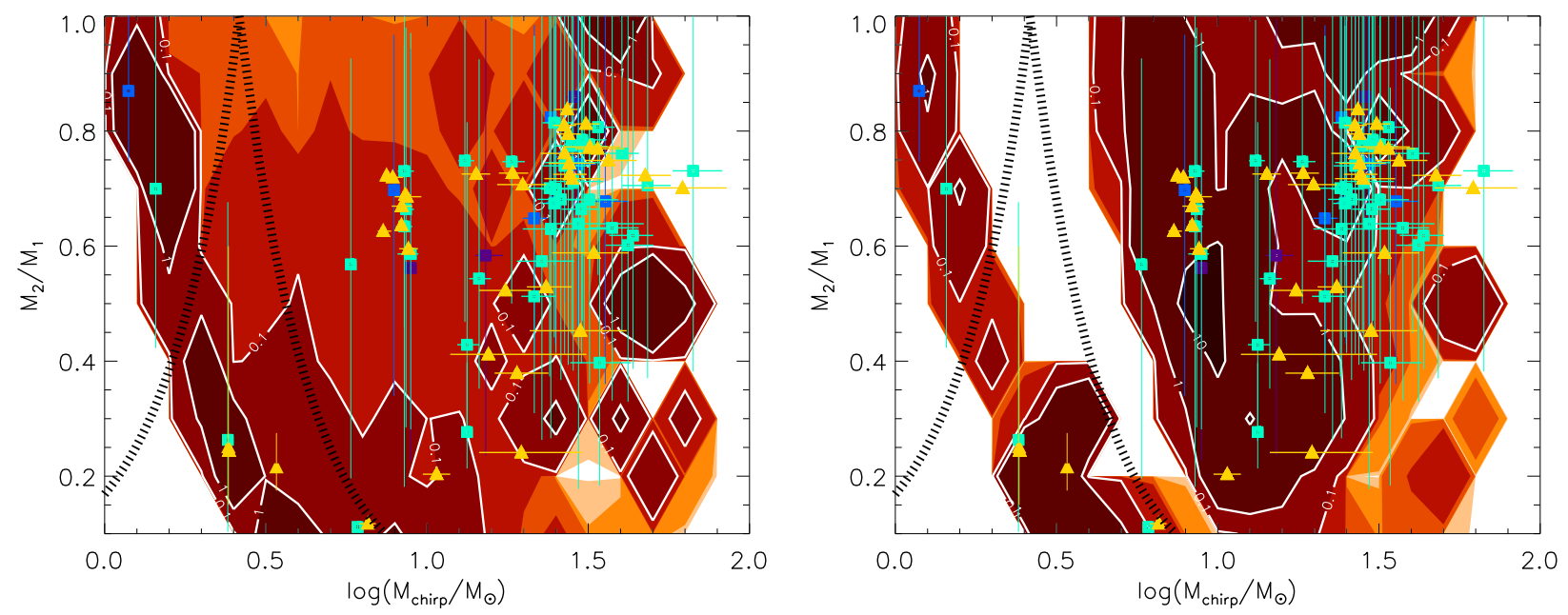

Figure A5. Details as in Fig. 3 but here the LHS panel shows the Standard remnant mass prescription and the Bray kick while the RHS shows the FryerRapid remnant mass prescription and the Hobbs kick
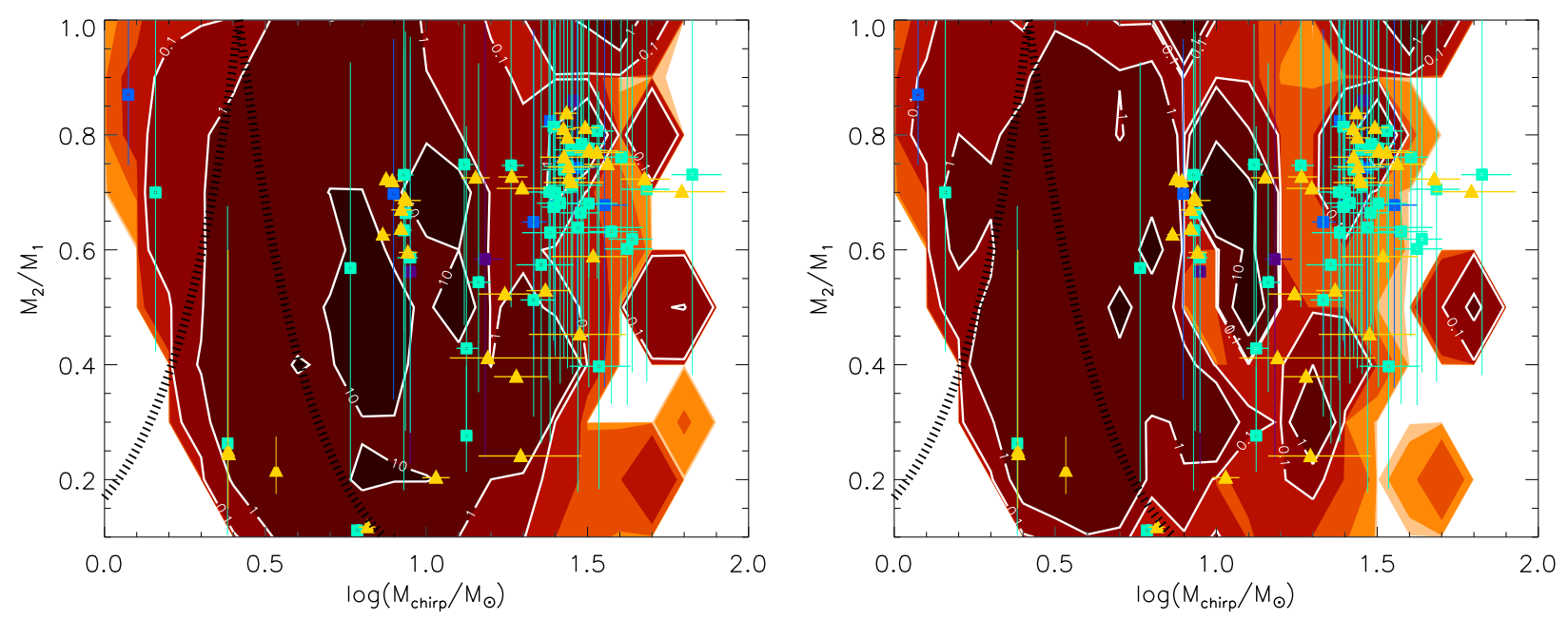

Figure A6. Details as in Fig. 3 but here the remnant mass is estimated from the $\mathrm{M}_{\mathrm{CO}}$, final remnant mass prescription with the LHS showing the Hobbs kick and the RHS the Bray kick. The mergers are biased towards the mid-range of the $\mathrm{M}_{\text {chirp }}$ values, providing a poor match to the observed data. 